\title{
Il Morgagni tra il Malpighi e il Cotugno
}

Dall'anatomia microscopica (Malpighi) all'anatomia patologica (Morgagni) e verso la istologia patologica (Cotugno)

di Luigi Belloni

Ringrazio la Società Svizzera di Storia della Medicina e delle Scienze Naturali, per avermi affidato la lettura Guggenheim 1981 ${ }^{1}$, sottolineando con ciò una collaborazione che risale al 1945. Ho volentieri accettato la proposta di parlare in italiano fattami dal Presidente Prof. H. Koelbing, soprattutto perchè fosse presente nella serie delle letture anche la terza lingua confederata.

L'argomento verte sulle avventure dei primi due secoli del microscopio nell'ambito della strutturistica animale. È un fondamentale capitolo del c. d. «romanzo del microscopio»: titolo che avrei potuto anche proporre per questa lettura, se non ne avessi temuto il sapore troppo giornalistico.

Nel nostro discorso, per quanto stringato ${ }^{2}$, prenderemo in considerazione le conquiste del microscopio, sia dirette, sia indirette, intendendo per tali quelle derivate dalla nuova mentalità indotta dalla introduzione dello strumento nella ricerca anatomica.

Come è ben noto, l'ingrandimento ottico potenziato mediante combinazione empirica di lenti, assurse a dignità di strumento scientifico, allorchè Galileo Galilei (1564-1642) puntò al cielo notturno stellato l'«occhiale» per l'osservazione «da lontano», e compì quella nutrita serie di fondamentali scoperte celesti che tosto descrisse nel Sidereus Nuncius (1610). I satelliti di Giove erano autentiche scoperte: la regolarità matematica con cui apparivano e scomparivano, escludeva la possibilità di una illusione ottica, che, come vedremo, sarà insidia costante dell'ingrandimento ottico.

Grazie all' «occhiale», l'atomismo sembrò concretarsi in dati di fatto e spogliarsi del carattere astratto proprio delle speculazioni filosofiche.

Non intendo ripetere quanto ho già esposto ${ }^{3}$ sulla osservazione microscopica della superficie esterna degli insetti e in particolare sulla Melissographia (1625) dell'Accademia dei Lincei. Mi limito a sottolineare che l'ingrandimento ottico esercitò indubbiamente una parte importante nella rinascita della filosofia democritea e della dottrina atomistica verificatasi nel XVII secolo. Significativa è l'affermazione di Francis Bacon (1561-1626) nel 
Novum organum (1620): «... quale Perspicillum [il microscopio] si vidisset Democritus, exiluisset forte, et modum videndi Atomum (quem ille invisibile omnino affirmavit) inventum fuisse putasset» ${ }^{4}$.

Altrettanto significativo è il programma di progressiva atomizzazione del nostro organismo proposta da Marco Aurelio Severino (1580-1656) ${ }^{5}$ nella Zootomia Democritaea (1645) tramite la «anatomia artificiosa et subtilis».

La microscopia promette di svelare le strutture minute nascoste nell'intimità dei nostri organi; e con le sue prime conquiste non soltanto promosse nei ricercatori una mentalità micrologica, ma consentì loro di riconoscere al tempo stesso una micrologia della natura, espressione che troviamo usata nella introduzione al De viscerum structura (1666) di Marcello Malpighi (1628-94), che fu per l'appunto il fondatore dell'anatomia microscopica.

La vicenda del Malpighi è ben nota: e può essere facilmente seguita nel volume delle sue Opere scelte citato alla Nota 2.

Molto importante fu l'anno 1656 , in cui, chiamato alla cattedra di medicina teorica all'Università di Pisa, il Malpighi si incontrò con Giovanni Alfonso Borelli (1608-79), chiamato invece alla cattedra di matematiche.

Allievo, tramite Benedetto Castelli (1577-1643), del Galilei, il Borelli dedicò gli ultimi decenni della sua vita alla stesura del De motu animalium (Roma 1680/81), breviario della iatromeccanica, per cui il metodo fisicomatematico proprio di Galileo e della sua Scuola trovò sistematica applicazione dal mondo inorganico a quello vivente.

Fondamentale nella evoluzione del Malpighi fu la collaborazione da lui intrattenuta col Borelli: «Egli adunque si compiaque instradarmi nello studio della filosofia libera e democritica, e da esso riconosco ciò che

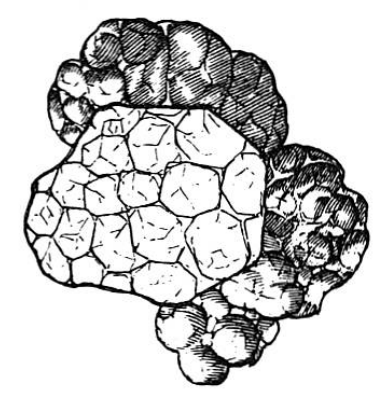

Fig. I. Frustolo di polmone in cui risaltano le vescicole polmonari del Malpighi, ossia i nostri alveoli. Da De pulmonibus observationes anatomicae (Bologna 1661) di M. Malpighi 


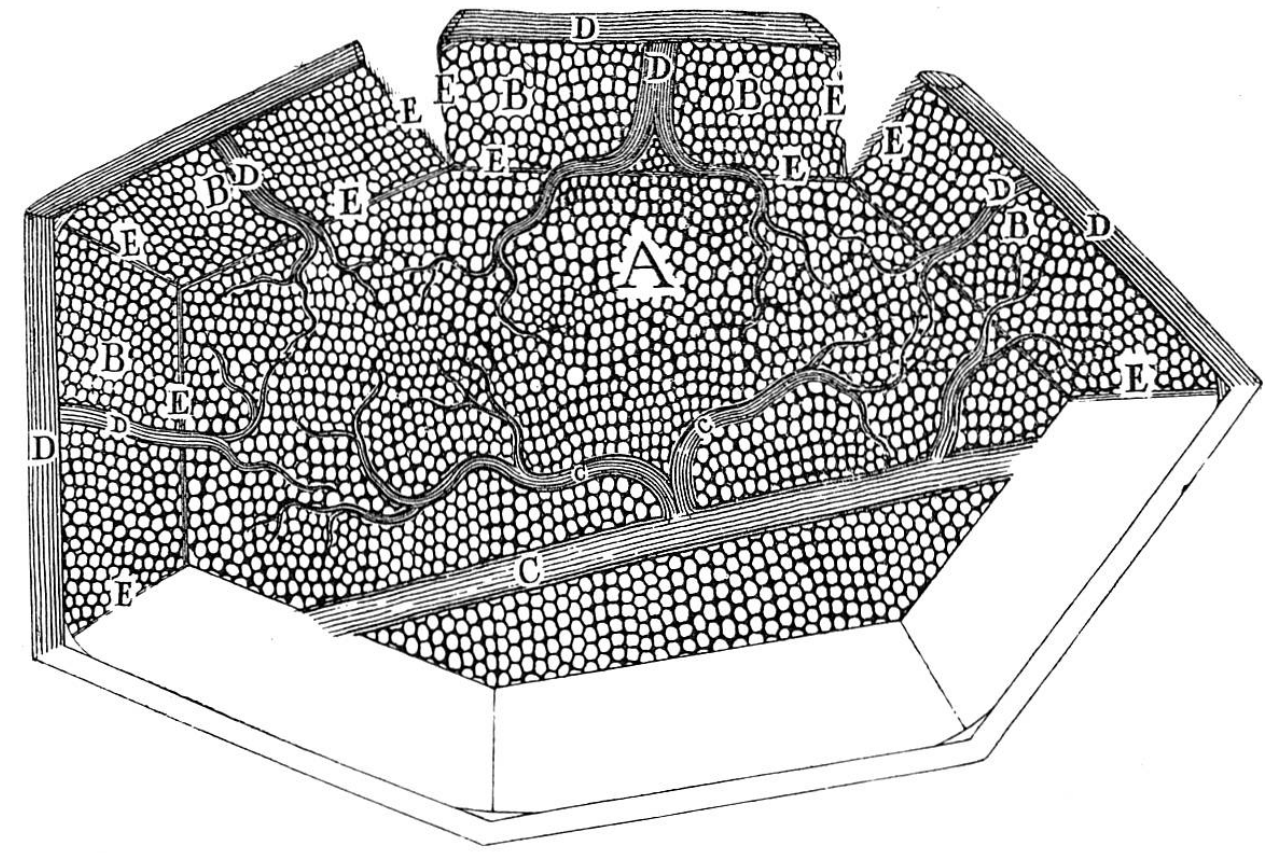

Fig. II. Celletta di un sacco polmonare di rana con la rappresentazione della rete capillare (A) interposta fra le ultime diramazioni dell'arteria (C) e della vena (D). Da De pulmonibus epistola altera (Bologna 1661) di M. Malpighi

d'avanzamento io ho fatto filosofando.» In altri termini, il Malpighi, formato a Bologna nella filosofia peripatetica, abbracciò, grazie al Borelli, la metodologia scientifica Galileiana.

Nella casa-laboratorio del Borelli, strutture come le fibre spirali del miocardio e i tubuli seminiferi dei testicoli ${ }^{6}$, furono scoperte mediante la sola «anatomia artificiosa et subtilis» senza l'aiuto del microscopio. L'addestramento così acquisito nell'artificio anatomico fu fondamentale per il Malpighi, che nel 1660, rientrato da Pisa a Bologna, imparò ad accoppiarlo con l'osservazione microscopica, e affinando sempre più la collaborazione fra mani e occhi, compì quella mirabile serie di scoperte che fanno di lui il fondatore dell'anatomia microscopica.

Alle scoperte sui polmoni - alveoli polmonari (Fig. I), ma soprattutto capillari sanguigni (Fig. II) - altre ne seguirono sulla struttura della lingua e della cute.

Principale artificio anatomico da lui adottato per queste ricerche fu la scomposizione in strati della parte superficiale della lingua, previa cottura. Diversamente dalla cuoca che «spella» la lingua bollita asportando un solo involucro, il Malpighi riuscì a scomporre i due strati di cui tale involucro si 
compone. In questa lingua (Figg. 1 e 2), ripetendo l'artificio anatomico del Malpighi ${ }^{7}$, abbiamo ottenuto per scomposizione i seguenti strati dalla superficie alla profondità (Fig. 2):

a) lo strato corneo, spesso e fornito di cornetti (per la compressione delle ghiandole salivari del palato)

b) lo strato reticolare che porta tuttora il nome del Malpighi, detto anche strato mucoso o gelatinoso. Verso l'esterno, i fori della rete sono abitati dalle radici dei cornetti, esse pure asportate assieme allo strato corneo.

c) il corpo papillare, da cui promanano le papille (di $1^{\circ}, 2^{\circ} \mathrm{e} 3^{\circ}$ ordine) concepite dal Malpighi come recettori gustativi, in quanto stimolabili dalle particelle dei corpi sapidi disciolte nella saliva e approfonditesi lungo i sottili canalini che egli riteneva attraversassero a pieno spessore gli strati corneo e reticolare. Così il Malpighi trovò un substrato anatomico per il meccanismo estesiologico previsto da Galileo ne Il Saggiatore (1623) ${ }^{8}$.

La ricerca Malpighiana dell'organo esterno del tatto parte dalla zampa del suino. Avulso, previa scottatura in acqua bollente, lo zoccolo, appare una foresta di «papille oblunghe e quasi piramidali (Fig. 3) emergenti a guisa di spade estratte dalle guaine»: e le guaine così svuotate corrispondono ai fori dello strato reticolare (Fig. 4), che in questa sede - e soprattutto nel talloneraggiunge uno spessore particolarmente notevole.

Fig. 1. Lingua di vitello sottoposta agli stessi artifici usati dal Malpighi (cottura e asportazione per slaminamento dello strato superficiale). La punta della lingua è rimasta integra onde appare tuttora irta dei cornetti di cui è dotata. Asportato dalla metà destra della lingua il solo strato corneo, è stato svelato lo strato reticolare o mucoso: è la c. d. rete del Malpighi, la quale salvo che nelle sfrangiature a bella posta sollevate - è rimasta in situ a ricoprire il corpo papillare. Nella figura si individua la sede del pigmento cutaneo nella parte profonda della rete

Fig. 2. Dettagli ingranditi della figura precedente. Dall'alto al basso: strato corneo, rete del Malpighi, derma con papille dei diversi ordini 

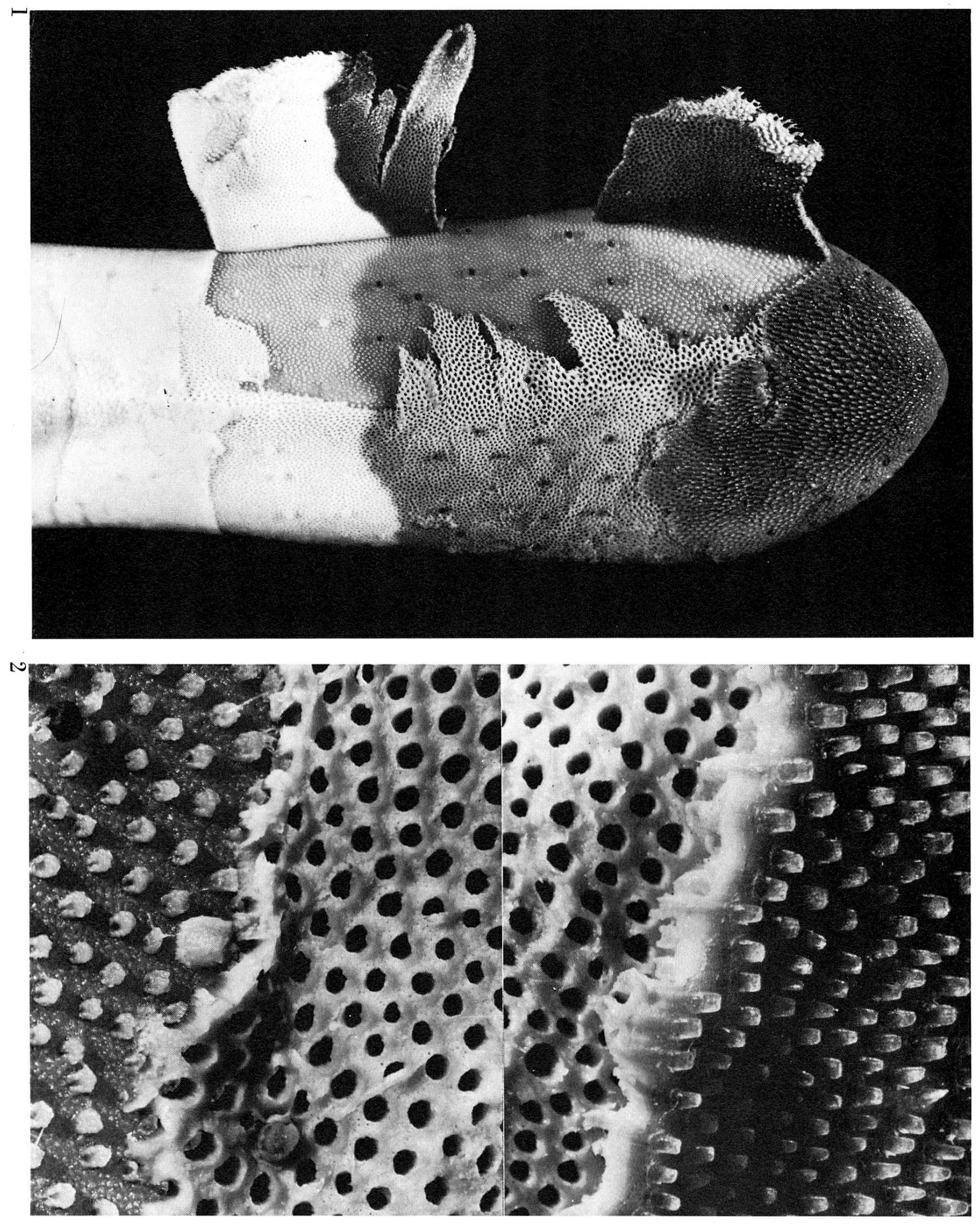


\section{Miliaris}

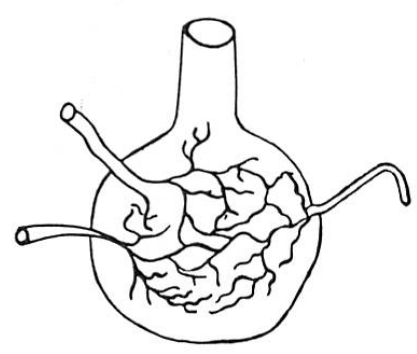

Fig.III. Autografo Malpighiano rappresentante lo schema strutturale della ghiandola elementare da lui propugnato: un follicolo che si continua nel tubo escretore ed è circondato dalle ultime diramazioni arteriose, venose e nervose

Anche nel «musello», del quale i quadrupedi «si servono per esplorare la terra e gli alimenti posti loro davanti», lo strato reticolare è molto alto (Fig. 5), ed è percorso a pieno spessore da lunghe ed esili papille, i cui apici arrivano a contatto col sottile strato corneo (cuticola) (Fig. 6).

Si tratta quindi di recettori tattili, che al pari dei recettori gustativi (o ritenuti tali) della lingua, sono concepiti dal Malpighi come estremità periferiche di fibre nervose. Sotto la cute del musello è poi evidente lo spesso strato di ghiandole elementari, di cui diremo (Fig.9 e Fig. III).

Figg. 3 e 4. Le due parti dell'ungula della zampa di suino scomposte mediante scottatura in acqua bollente al limite epitelio-derma. Le lunghe ed esili papille (Fig. 3) emergenti dal derma, appaiono come spade estratte dalle loro guaine, e precisamente dai forami dello strato reticolare (Fig. 4)

Fig. 5. Porzione di musello bovino scomposta col metodo della bollitura usato dal Malpighi. Dall'alto al basso: cuticola, strato Malpighiano, derma irto di papille

Fig. 6. Sezione istologica di musello a pieno spessore preparata secondo la tecnica odierna (colorazione ematossilina-eosina): lunghe papille dermiche attraversano a pieno spessore lo strato Malpighiano fino a raggiungere la cuticola 

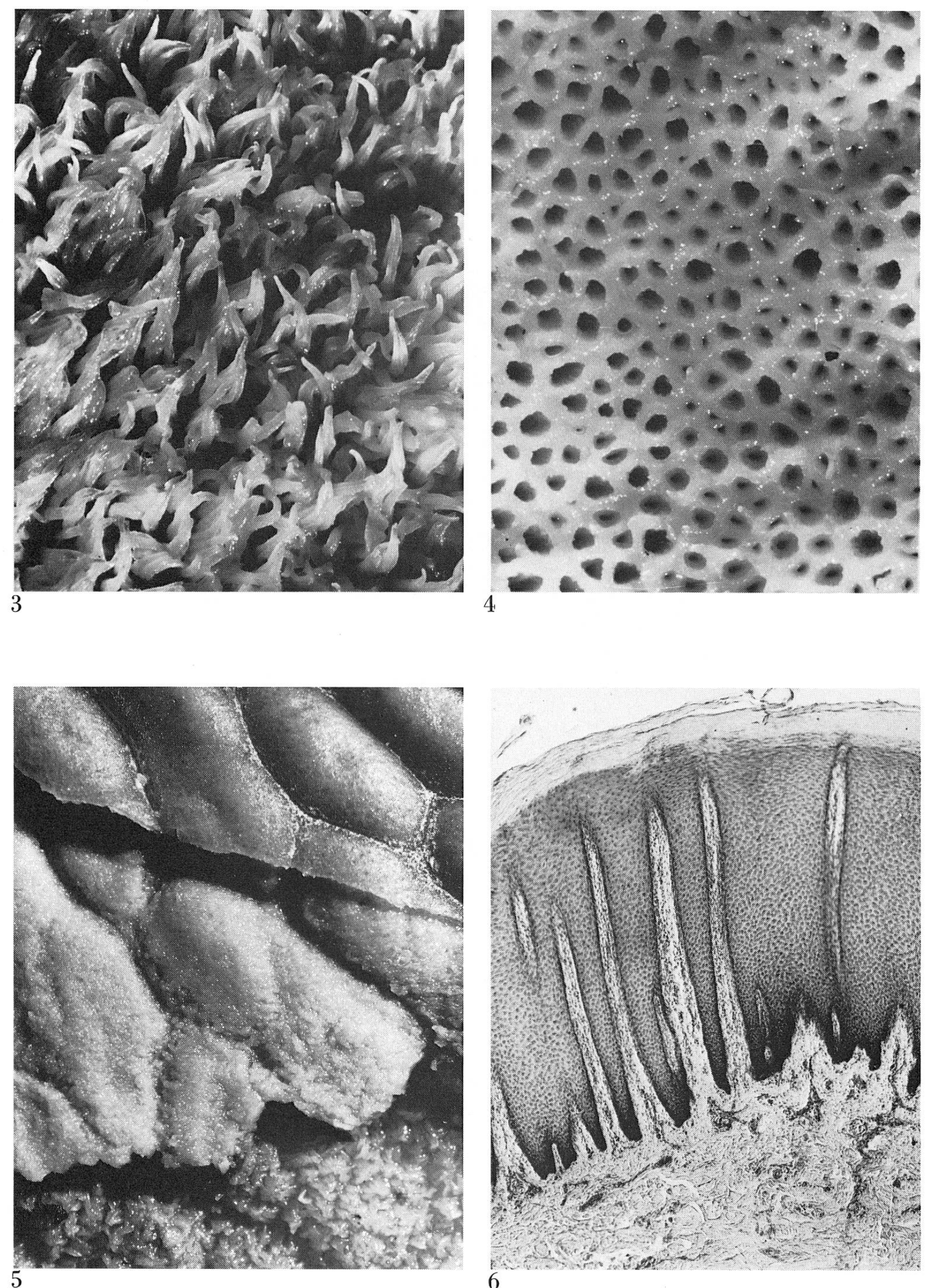
Finalmente è la volta del polpastrello delle dita con le sue rughe spirali, che, denudate grazie all'ustione con un ferro incandescente, appaiono costituite da due ordini paralleli di papille, intercalati dagli orifici dei vasi sudoriferi.

Altre macchine più complesse strutturò il Malpighi nel nostro organismo. Particolarmente complessa è ad es. la macchina della diuresi che sta a fondamento del nostro «nefrone», e fu descritta dal Malpighi nel De renibus (1666). Sulla superficie di sezione della corticale (Fig. 7), emergono, colorati per affusione di inchiostro, tubuli, in parte retti e in parte contorti; e colorati per via endoarteriosa (Fig.8), ciuffi capillari, appesi ai ramoscelli delle arterie interlobulari. Sono i futuri glomeruli: e le loro anse (Fig. 8a) sembrano fatte a bella posta per abbracciare l'estremità ampollare del tubulo (Fig. 7 a) ${ }^{9}$, concepita come follicolo ghiandolare. Le immagini così ottenute sembrano particolarmente convincenti per strutturare la macchina della diuresi sullo schema della ghiandola elementare, il quale ne esce collaudato su di un piano microscopico, per i tempi particolarmente fine. Secondo il Malpighi, infatti, la ghiandola elementare, o miliare (Fig. III), si compone sostanzialmente di un follicolo membranoso secernente - attorno al quale si sfioccano le arterie, le vene e i nervi - e di un tubulo escretore, che rappresenta la continuazione del follicolo ${ }^{10}$.

Fig. 7. Rene di capretto: superficie di sezione cosparsa con inchiostro diluito e tosto asciugata. Viene così colorato soltanto l'interstizio, il quale mette pertanto in risalto le strutture parenchimali (tubuli retti e contorti) non impregnate dall'inchiostro

Fig. 8. Rene di capretto: sezione longitudinale mediana della corticale, previa iniezione di inchiostro di china diluito nell'arteria renale. Spiccano così i tronchicini delle arterie interlobulari coi loro rami, ai quali sono appesi, a mo' di pomi, i corpuscoli del Malpighi

Fig. 7 a. Estremità ampollare di un tubulo renale (dettaglio della figura 7)

Fig. 8 a. Glomerulo renale del Malpighi con le due arteriole (afferente ed efferente) (dettaglio della figura 8) 


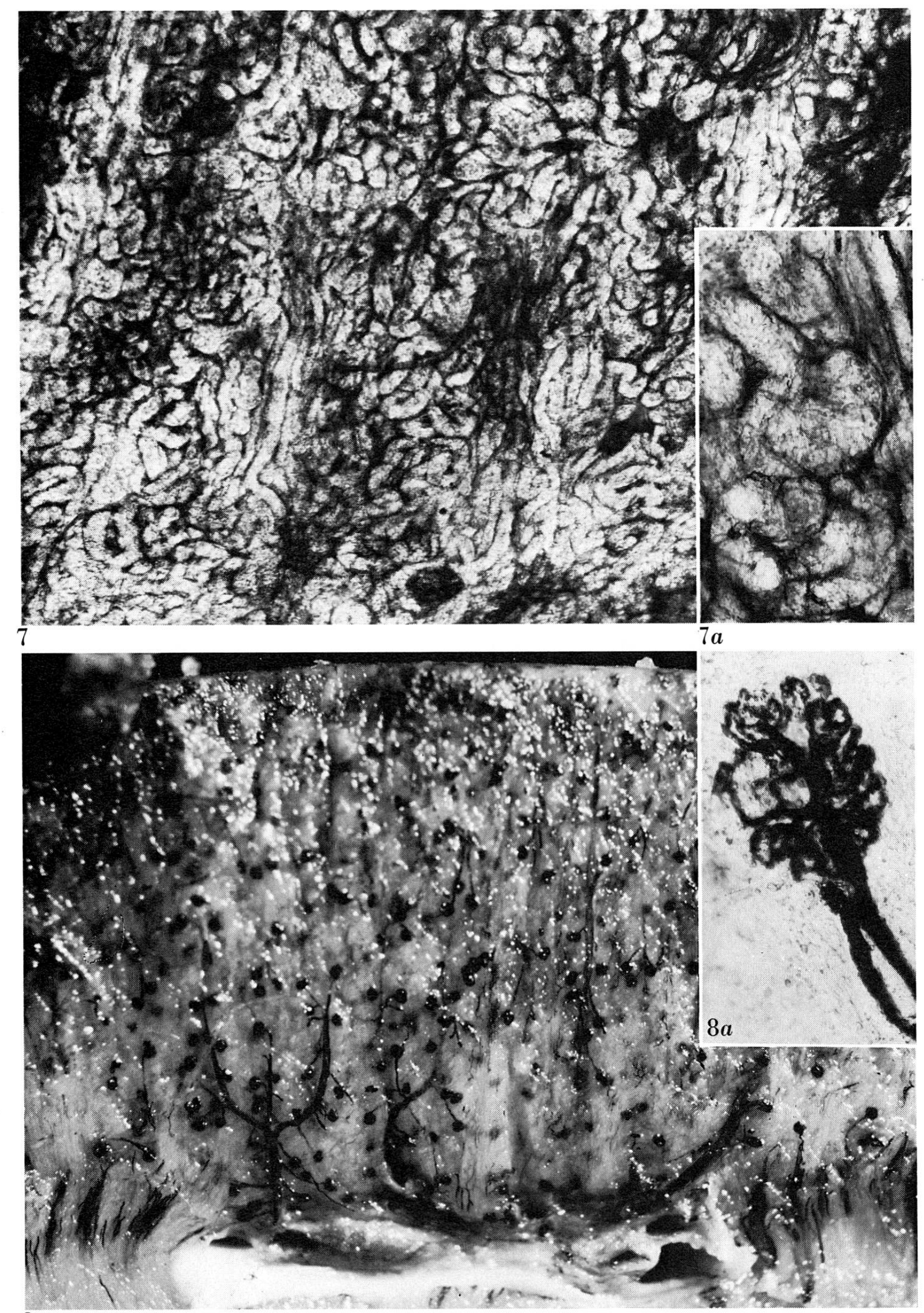


Gli allievi del Malpighi cercarono de seguirne le orme. Anche se non raggiunsero risultati paragonabili ai suoi, e se usarono ingrandimenti assai più modesti, essi manifestarono tuttavia la sua stessa impostazione iatromeccanica. Cito ad es. le «cordelline sonore» dell'orecchio interno messe in evidenza da Anton Maria Valsalva (1666-1723) nel De aure humana (1704), e interpretate come il recettore acustico meccanicamente stimolabile dal «frequente tremor dell'aria in minutissime onde increspata». ${ }^{11}$

Assai più importante degli allievi diretti del Malpighi fu però il loro allievo Giovanni Battista Morgagni (1682-1771) ${ }^{12}$. Da suoi spunti autobiografici risulta che, dopo aver difeso quattordicenne in Forlì, sua città natale, la filosofia peripatetica, egli si trasferì nel 1698 a Bologna, dove «studiò la moderna Filosofia e la Medicina, nelle quali ebbe per Maestri vari celebri Allievi del Malpighi: il Sandri, l'Albertini, il Valsalva». Non si ripete forse in queste righe la conversione del Malpighi, che giunto peripatetico da Bologna a Pisa, fu convertito dal Borelli alla filosofia Galileiana? Del Morgagni potremo considerare soltanto il capolavoro: il De sedibus et causis morborum per anatomen indagatis (1761), che fu il «Lebenswerk» di un ottantenne che aveva mantenuto pienamente integra la sua eccezionale lucidità di mente.

Il De sedibus parte, per l'appunto, da un motivo fornito dal Malpighi, che il Morgagni seppe sviluppare in un'opera grandiosa: ed è un motivo che può essere formulato in termini estremamente semplici. Il nostro organismo è un complesso di macchine. La vita è la resultante del funzionamento armonico di queste macchine, molte delle quali, particolarmente delicate e minute, come quella della diuresi, sono state scoperte dal microscopio nella intimità degli organi, in cui si trovano nascoste. Al pari di quelle inorganiche, anche le macchine organiche sono soggette a deterioramenti, ad avarie, a guasti, che ne alterano il funzionamento. Questi guasti incidono ai livelli più minuti delle macchine organiche; ma, per ragioni che vedremo, sono suscettibili di uno studio attendibile soltanto al livello macroscopico, attraverso l'indagine delle lesioni d'organo al tavolo anatomico. Le alterazioni funzionali che derivano da questi guasti, producono sconcerti nell'economia dell'organismo, che si traducono in manifestazioni cliniche proporzionate alla sede e alla natura dei guasti stessi.

È il programma implicito nel titolo stesso dell'opera del Morgagni De sedibus et causis morborum per anatomen indagatis. Un guasto in un punto del complesso meccanico dell'organismo, indagato mediante l'anatomia, è la sede e la causa della malattia, o meglio, dei fenomeni clinici di questa, i quali sono concepibili come alterazioni funzionali derivate dal guasto medesimo. 


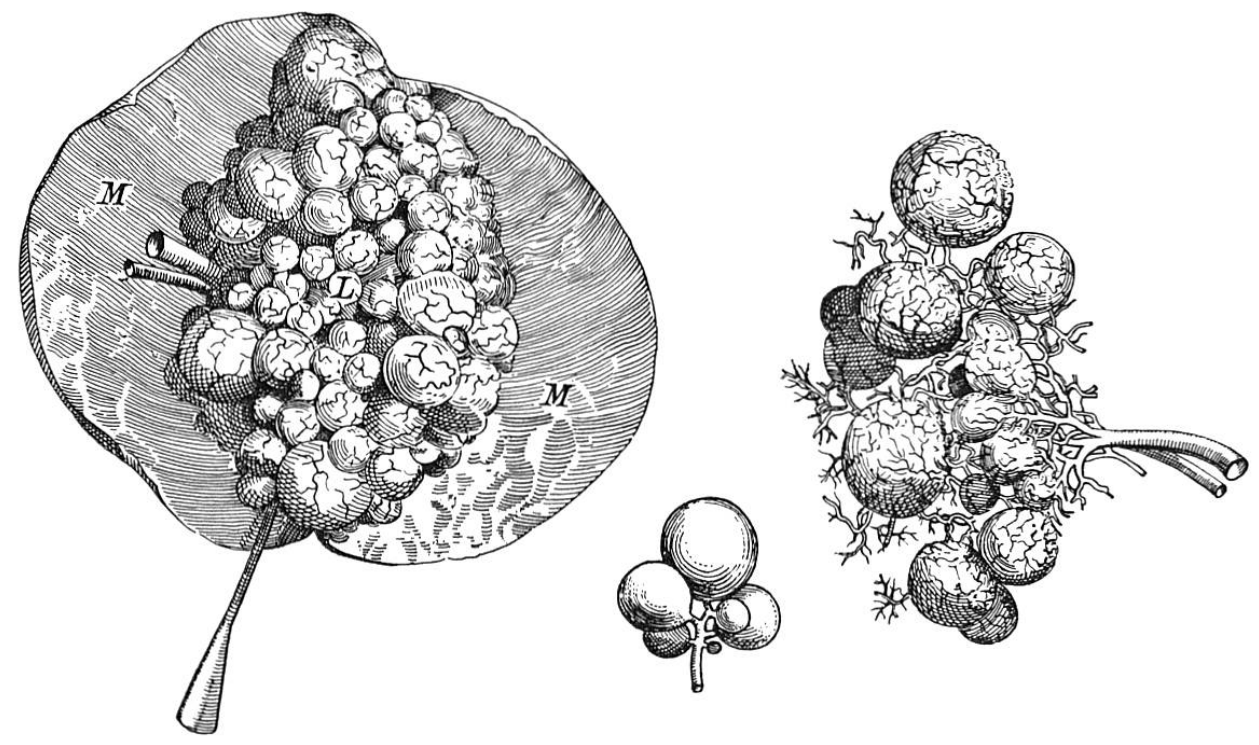

Fig. IV. Il rene policistico descritto nel 1705 da Alexis Littre (1658-1726). Secondo la morfogenesi meccanica proposta dall'autore, i follicoli ghiandolari sono stati fortemente distesi dalla urostasi, provocata dalla occlusione ureterale (fig. a sinistra). Il rene ha pertanto assunto l'aspetto di un grappolo d'uva: i follicoli sono appesi, a mo' di acini, ai tubuli escretori (fig. intermedia), e sono inoltre abbracciati dalle diramazioni terminali dell'arteria, della vena e del nervo (fig. a destra)

Il parallelismo fra lesione anatomica e sintomo clinico qualifica le c.d. historiae anatomico-medicae, che sono i mattoni con cui il Morgagni costruì il De sedibus.

Raccolte casistiche di questo genere esistevano anche prima del Morgagni: e in particolare, il Sepulchretum (Ginevra, 1679 e 1700) di Théophile Bonet (1620-89); ma si tratta di una «compilation indigeste et incohérente», come dirà anche il Laennec ${ }^{13}$, sfornita dei pregi dell'opera Morgagnana, e in particolare del lavoro di coordinazione illuminato dalla diretta esperienza di un grande anatomico.

Nel De sedibus la fisiologia ha una parte fondamentale, in quanto essa fornisce al Morgagni gli elementi per risalire dal guasto della macchina alla sua alterazione funzionale. Ricordo che la fisiologia sperimentale trovò proprio allora la sua grande sintesi negli Elementa physiologiae corporis humani di Albrecht von Haller (1708-1777), apparsi contemporaneamente (1757-1766) al De sedibus.

L'opera del Morgagni è frutto di continui esami comparativi: fra due o più reperti anatomici affini, come pure fra sintomi clinici affini, oltre che fra i due ordini di fenomeni. È una serie continua di legami che vengono così stabiliti: 
è un lavoro ininterrotto di coordinazione, di sintassi, che sfrutta molto abilmente lo strumento prezioso degli indici, il cui ausilio è pienamente valorizzato dallo stesso Morgagni nella prefazione.

Al pari degli Elementa physiologiae del von Haller, anche il De sedibus del Morgagni è una grandiosa opera di sintesi, che portò al riconoscimento dell'anatomia patologica quale parte integrante della medicina e quale premessa per il suo ulteriore sviluppo: alludo in particolare agli sviluppi della clinica nella Parigi dei primi decenni del XIX secolo.

Fig. 9. Musello bovino scottato in acqua bollente e spaccato secondo il piano longitudinale mediano. Sotto la cute è riconoscibile lo strato ghiandolare coi suoi acini, i quali ricevono dalla profondità vasellini sanguigni, nonchè alcune propaggini filamentose interpretate dal Malpighi come fibre nervose. Dal polo opposto degli acini partono i condotti escretori, messi in evidenza dalla intensa pigmentazione, e si dirigono verso l'epitelio fino a sboccare alla superficie cutanea. Questi e analoghi reperti (Figg. $7 a$ e $8 a$ ) stanno alla base dello schema Malpighiano della ghiandola elementare (Fig. III)

Fig. 10. Dettaglio di una lamina smalto-dentinale di molare non ancora erutto di vitello osservata a luce trasmessa. La superficie esterna dello smalto è rivolta verso l'obiettivo; e la superficie profonda della dentina, verso il tavolino del microscopio. Appaiono i prismi dello smalto col loro decorso ondulato, sovrapponibile al disegno del Malpighi (Anatome plantarum, Londra, vol. 1, 1675, pp.19-20 e Fig. 37)

Fig. 11. Osservando a luce riflessa lo stesso campo senza variazioni di ingrandimento, i prismi vengono adombrati dalla immagine illusoria «a strie di peli elevantisi», che è meglio evidente man mano diminuisce l'ampiezza dei prismi. Si tratta di un effetto ottico riferibile alle ondulazioni dei prismi dello smalto e responsabile delle c.d. strie di Hunter-Schreger visibili anche ad occhio nudo

Fig.12. La struttura fibrillare dell'organismo nella Pharmaceutice rationalis (Londra 1674/75) di Thomas Willis (1621-1675)

Fig. 13. L'immagine illusoria filamentoso-reticolare osservata nel 1960 da Bruno Zanobio con microscopi non acromatizzati del $18^{\circ}$ secolo

Fig. 14. L'immagine illusoria filamentoso-reticolare indusse Felice Fontana (1730-1805) a considerare i cilindri tortuosi quale struttura elementare dell'organismo

Fig. 15. La stessa immagine illusoria indusse Henri Milne Edwards (1800-1885) ad attribuire all'organismo una struttura elementare globulare. La dottrina globulare, frutto, come si è detto, di una illusione, precorse pertanto la dottrina cellulare 


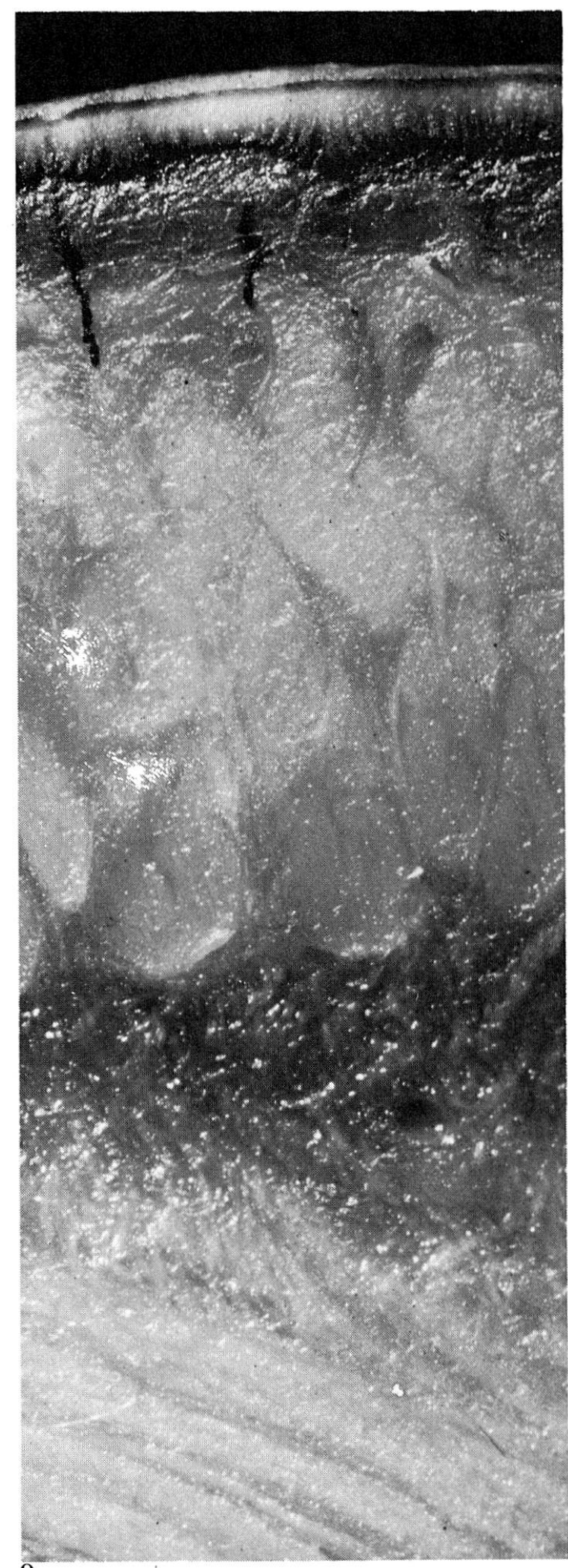

9

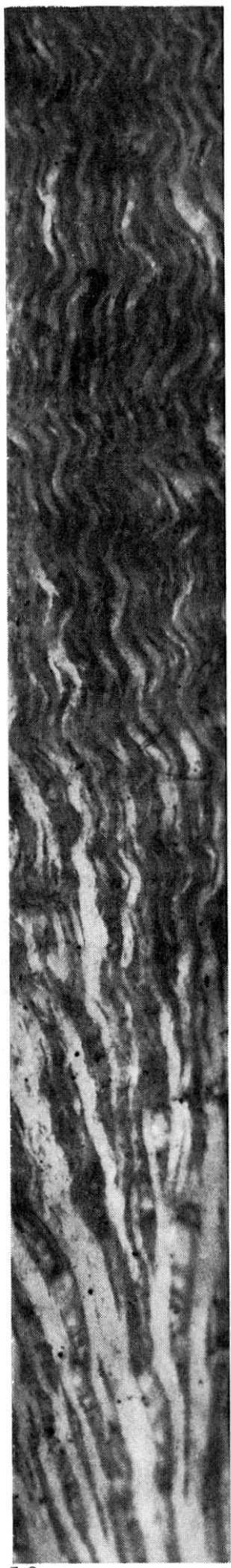

10

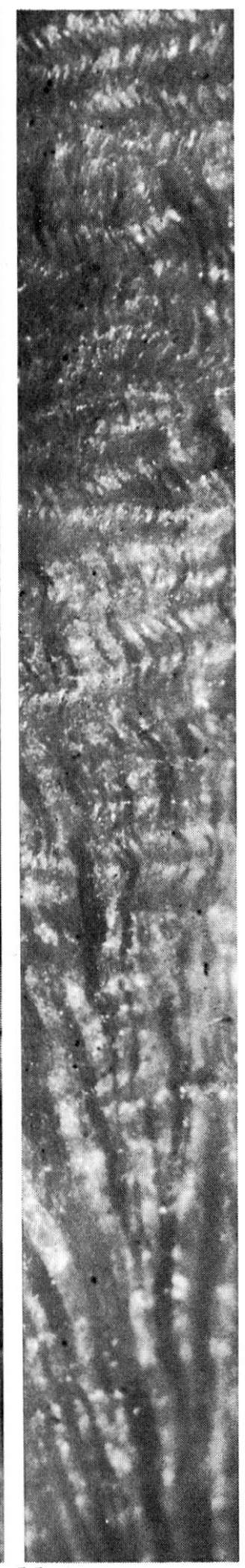

11

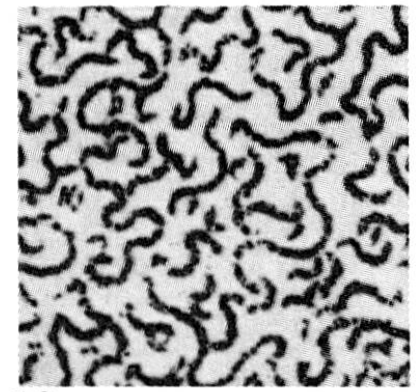

12

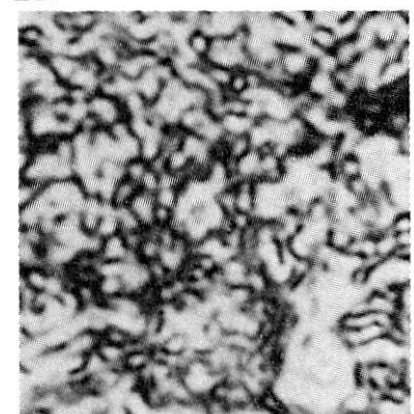

5) 340 Heg हुा

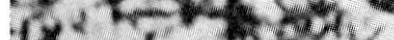

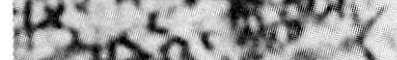

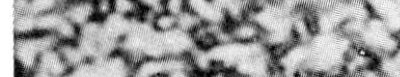
2. $3{ }^{2} \times \mathrm{x}^{2}$

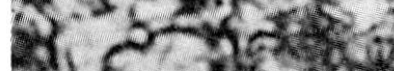

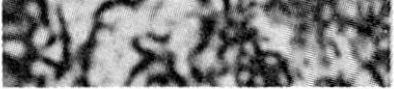
13

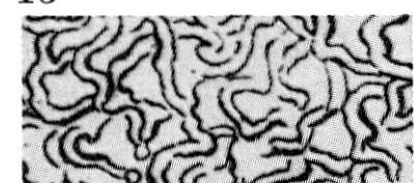
9. 13 है पrop की जाड़क 14

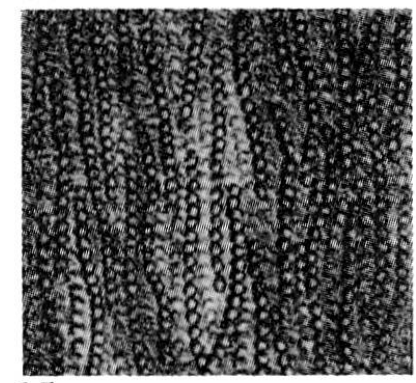

15 
A questo punto si pone un importante problema: come mai l'anatomia patologica del Morgagni è puramente macroscopica? Eppure sappiamo che l'anatomia microscopica aveva avuto una parte notevolissima nella sua nascita.

Presento ad es. (Fig. IV) questo rene policistico congenito bilaterale descritto nel 1705 da Alexis Littre (1658-1726). Il reperto è puramente macroscopico: eppure fu spiegato dal Littre, come d'altronde aveva fatto già il Malpighi ${ }^{14}$ nel 1674 per casi analoghi, invocando la struttura microscopica del rene: «Ho quindi sospettato che fossero ghiandole miliari dilatate e turgide di urina, la quale non avendo potuto uscire attraverso un proprio vaso escretore, era ristagnata» nel follicolo.

Ritorno, quindi, al problema iniziale. Perchè il microscopio, dopo scoperte così brillanti compiute nel campo dell'anatomia normale durante il XVII secolo, fu ignorato dall'anatomia patologica, che si sviluppò sopra tutto nel secolo successivo? Fondamentale in questo senso fu l'inibizione esercitata dalle illusioni ottiche.

Il Malpighi, per quanto mi è riuscito di documentare, cadde vittima di una sola illusione ottica, laddove descrisse i prismi dello smalto dentario ritenendoli formati da «strie di peli elevantisi». ${ }^{15}$ Proviamo ad osservare a modesto ingrandimento una lamina smalto-dentinale di un molare non erutto asportato da una mandibola di vitello. Ecco i prismi dello smalto osservati a luce trasmessa (Fig. 10). Ed ecco lo stesso preciso campo osservato a luce riflessa (Fig. 11): si ha l'impressione di «strie di peli elevantisi» quali si osservano nel velluto schiacciato. È una illusione ottica dovuta all'ondulazione dei prismi e nota anche per i tronchicini nervosi ondulati ${ }^{16}$.

Allo stato attuale delle mie ricerche, penso di poter escludere per il Malpighi un'altra illusione che, già reperibile in qualche ricercatore dei suoi tempi-Willis (Fig. 12), Kerckring - divenne sempre più frequente nel XVIII secolo e nei primi decenni del XIX. È la immagine filamentoso-reticolare ${ }^{17}$ generosamente prodotta dai microscopi composti non ancora acromatizzati di quel periodo: una immagine sicuramente illusoria (Fig. 13), poiché rimane costante, senza sostanziali variazioni, qualunque sia la parte dell'organismo sottoposta all'obiettivo del microscopio.

Diversamente da molti altri ricercatori dell'epoca, Alexander Monro sec. (1733-1817), Felice Fontana (1730-1805) (Fig.14), e più tardi Paolo Mascagni (1752-1815), il Morgagni rifuggì per la sua anatomia patologica dall' impiego del microscopio composto, appunto per non cadere nella trappola delle immagini illusorie. 


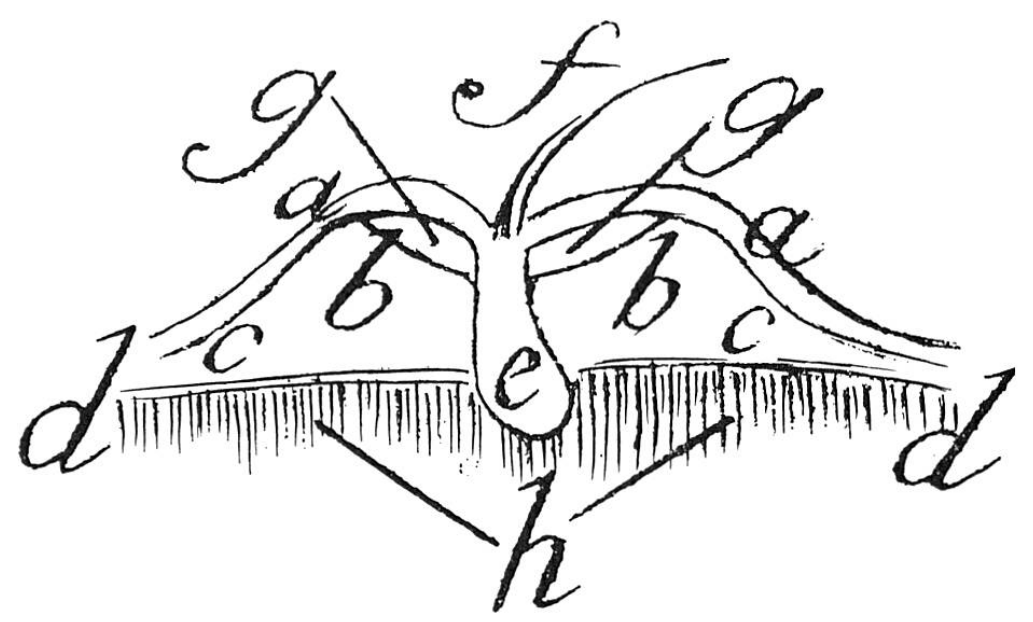

Fig. V. Schema di una pustola vaiuolosa sezionata trasversalmente (ingrandimento $5 \mathrm{x}$ ). Nel derma $d$ i vasi $h$ sono dilatati e congesti. La rete del Malpighi $b$ appare necrotica e tumefatta, e inizia a subire in $g$ la colliquazione purulenta. La sovrastante cuticola a presenta soltanto un lieve ispessimento. Dal De sedibus variolarum (Napoli 1769) di Domenico Cotugno

Assolutamente reciso contro l'uso del microscopio sarà oltre mezzo secolo più tardi un anatomo-patologo della forza di R.Th. Hyacinthe Laennec (1781-1826):

«... si l'on cherche les causes de maladies graves dans des altérations microscopiques des organes, il est impossible de ne pas tomber dans des conséquences absurdes, et l'anatomie pathologique, ainsi que celle de l'homme sain, cultivée de cette manière perdraient bientôt le rang qu'elles occupent parmi les sciences physiques, pour se changer en un vaste champ d'hypothèses fondées sur des illusions d'optique et des spéculations, sans aucune utilité réelle pour la médecine». ${ }^{18}$

In queste righe echeggia quel netto ripudio del microscopio affermato con tanta decisione da M.F.Xavier Bichat (1771-1802), fondatore della istologia $^{19}$ :

«... nos instruments microscopiques, espèce d'agents dont la physiologie et l'anatomie ne me paroissent pas d'ailleurs avoir jamais retiré un grand secours, parce que quand on regarde dans l'obscurité, chacun voit à sa manière et suivant qu'il est affecté». ${ }^{20}$

L'istologia del Bichat è in altre parole una istologia macroscopica. Il Bichat preparava i suoi tessuti, le membrane, mediante la scomposizione anatomica in cui già il Malpighi si era dimostrato maestro. Nuovamente proietto la lingua di vitello coi suoi tre strati (Fig. 2): cuticola, strato reticolare, e derma con le sue papille. Richiamo inoltre la scomposizione del musello bovino in analoghi strati: cuticola, muco Malpighiano, derma (Fig. 5). 
La conoscenza di questa sovrapposizione di strati permette di analizzare una lesione patologica quale è la pustola cutanea. La pustola vaiuolosa (Fig. V $)^{21}$, ad es., perde il carattere generico di «materia peccans» che deve essere eliminata dall'organismo. In seguito alla strutturazione della cute, essa assume la fisionomia di una alterazione complessa, quale associazione di lesioni peculiari a ciascun strato cutaneo. Nel derma $d$ i vasi $h$ sono dilatati e congesti; la rete del Malpighi $b$ appare necrotica e tumefatta: anzi la sua tumefazione è la principale responsabile del maggior volume della pustola rispetto alla cute circostante. In $g$ inizia la colliquazione purulenta del muco Malpighiano necrotico. La sovrastante cuticola $a$ presenta soltanto un lieve ispessimento.

Queste osservazioni di istopatologia macroscopica sono tratte dal $D e$ sedibus variolarum (Napoli 1769) di Domenico Cotugno (1736-1822), che, pur fiorito a Napoli, possiamo considerare come il maggior continuatore dell'opera del Morgagni in Italia. Le parole De sedibus che fungono da «incipit» ai titoli di entrambe le opere, quella del Morgagni (1761) e, otto anni più tardi, quella del Cotugno (1769), subiscono un ampliamento nella seconda opera: al localismo d'organo si sovrappone il localismo di tessuto. Già nel De ischiade nervosa (Napoli 1764) il Cotugno aveva distinto le alterazioni delle fibre nervose da quelle del tessuto interstiziale fra esse interposto. Sono questi esempi precorritori della necessità affermata dal Bichat «de considérer les maladies locales, non point sous le rapport des organes composés, qu'elles ne frappent presque jamais en totalité, mais sous celui de leurs tissus divers qu'elles attaquent presque toujours isolément».22

In conclusione, questa lettura si è proposta di sottolineare l'importanza del microscopio, non soltanto come strumento di osservazione diretta, ma anche per aver determinato la formazione di una mentalità micrologica $\mathrm{e}$ contribuito alla rinascita dell'atomismo. L'osservazione microscopica associata alla scomposizione anatomica diede nascita all'anatomia microscopica (Malpighi 1660), che, fecondata dalla iatromeccanica, mise in evidenza una serie di macchine organiche nascoste nella intimità degli organi. Si pose così il problema se anche le macchine organiche non potessero subire deterioramenti, avarie e guasti, al pari delle macchine inorganiche. Ne derivarono la indagine anatomica delle lesioni d'organo e l'analisi del rapporto con l'alterazione funzionale che ne deriva, ossia con la malattia clinica. Queste storie clinico-anatomiche («historiae anatomico-medicae») furono raccolte e criticamente valutate nel De sedibus et causis morborum per anatomen indagatis (1761) del Morgagni. L'anatomia patologica, per quanto ispirata 
dall'anatomia microscopica, rimase puramente macroscopica per non cadere vittima delle illusioni ottiche prodotte dai microscopi dell'epoca. Il microscopio fu altrettanto bandito dalla istologia normale e patologica, quale venne formulata dal Bichat, anche se l'analisi strutturale dell'anatomia microscopica aveva contribuito a prepararle la strada. Tipico è il caso della pustola vaiuolosa quale fu patologicamente strutturata nel 1769 dal Cotugno, il quale mise in evidenza lesioni diverse per ciascuno dei tre strati che erano stati riconosciuti un secolo prima dal Malpighi nella cute.

Note

${ }^{1}$ Dr. Markus-Guggenheim-Schnurr-Gedenkvortrag letto a Davos il 25 Settembre 1981 in occasione della riunione annuale della Società Svizzera di Storia della Medicina e delle Scienze Naturali.

${ }^{2}$ Per il quale mi rifaccio alla introduzione alle Opere scelte del Malpighi da me curate per la collana «Classici della Scienza» della Utet (Torino 1967). L'introduzione è corredata dalla bibliografia dei precedenti lavori dei miei Collaboratori e miei sulla storia della microscopia.

${ }^{3}$ Luigi Belloni, Il Primo Ventennio della Microscopia (Galilei 1610 - Harvey 1628). Dalla Microscopia alla Anatomia Microscopica dell'Insetto, in: Clio Medica 4 (1969), pp. 179-190. Consiglierei anche, se non fosse difficile da reperire, Luigi Belloni, Il microscopio applicato alla biologia da Galileo e dalla sua Scuola (1610-1661), in: Saggi su Galileo Galilei, raccolti e pubblicati a cura di Carlo Maccagni, Vol. III, Tomo 2, delle Pubblicazioni del Comitato Nazionale per le Manifestazioni Celebrative, Firenze 1972, pp.689-730.

${ }^{4}$ II 39.

${ }^{5}$ Luigi Belloni, Severinus als Vorläufer Malpighis, in: Nova Acta Leopoldina 27 (1963), n. 167, pp. 213-224.

${ }^{6}$ Scoperti dal lorenese Claudius Aubrius, lettore di Anatomia nello Studio di Pisa. Cf. Luigi Belloni, Dall'«occhiale» di Galileo all'anatomia microscopica di M.Malpighi: il «Testis examinatus> (Firenze 1658) di Claudius Auberius, in: Rendiconti dell' Istituto Lombardo Classe di Scienze (B) 98 (1964), pp.205-226; Claudius Aubrius a Francesco M. Fiorentini sull'assunzione di latte da parte del feto nell'utero, in: Physis 19 (1977), pp. 377-382.

${ }^{7}$ In più punti, il testo non illustrato (o insufficientemente illustrato) del Malpighi riesce incomprensibile senza ripeterne l'artificio anatomico per riottenere il preparato da lui descritto. Cfr. Luigi Belloni, The Repetition of Experiments and Observations: Its Value in Studying the History of Medicine (and Science), in: Journal of the History of Medicine and Allied Sciences 25 (1970), pp. 158-167.

Questo metodo è stato usato anche recentemente, e con profitto, dal mio ex-allievo Renato G. Mazzolini per chiarire le ricerche compiute nel XVIII secolo sulla struttura dell'iride (The iris in the eighteenth-century physiology, Bern 1980).

8 «E perchè di questi corpi alcuni si vanno continuamente risolvendo in particelle minime, delle quali altre, come più gravi dell'aria, scendono al basso, ed altre, più leggieri, salgono ad alto; di qui forse nascono due altri sensi, mentre quelle vanno a ferire due parti del corpo nostro assai più sensitive della nostra pelle, che non sente l'incursioni di materie tanto sottili 
tenui e cedenti: e quei minimi che scendono, ricevuti sopra la parte superiore della lingua, penetrando, mescolati colla sua umidità, la sua sostanza, arrecano i sapori, soavi o ingrati, secondo la diversità de' toccamenti delle diverse figure d'essi minimi, e secondo che sono pochi o molti, più o men veloci; gli altri, ch'ascendono, entrando per le narici, vanno a ferire in alcune mammillule che sono lo strumento dell'odorato, e quivi parimente son ricevuti i lor toccamenti e passaggi con nostro gusto o noia, secondo che le lor figure son queste o quelle, ed i lor movimenti, lenti o veloci, ed essi minimi, pochi o molti» (G. Galilei, Le opere, edizione nazionale, VI, p.349).

${ }^{9}$ La situazione del tubulo all'interno della capsula sarà riconosciuta nel $1842 \mathrm{da}$ W. Bowman. Cf. Felice Grondona, La struttura dei reni da F. Ruysch a W.Bowman, in: Physis 7 (1965), pp.281-316. Luigi Belloni, La conquista del nefrone, in: Atti della Accademia Medica Lombarda 18 (1963), pp.911-929; Die Eroberung des Nephrons, in: Berliner Medizin 16 (1965) pp.92-100; La conquista del nefrone, in: Simposi Clinici 10 (1973), pp.XXV-XXXII; ristampato nel mio volume Per la Storia della Medicina, Bologna 1980, pp. 279-286.

${ }^{10}$ L.Belloni, Auf dem Wege zur Elementardrüse als Sekretionsmaschine. Forschungen des Kreises um Borelli (Auberius, Bellini-Zambeccari, Malpighi), in: Medizingeschichte im Spektrum. Steudel-Festschrift (= Beiheft 7 del «Sudhoffs Archiv»), Wiesbaden 1966, pp.11-29; Boerhaave et la doctrine de la glande, in G. A. Lindeboom (edit.) Boerhaave and his time, Leiden 1970, pp.69-82.

${ }^{11}$ L. Belloni, Suono e orecchio dal Galilei al Valsalva. (Nel terzo centenario della nascita di A.M.Valsalva), in: Simposi Clinici 3 (1966), pp.XXXIII-XLII, ristampato nel mio volume Per la Storia della Medicina, Bologna 1980, pp.69-78.

${ }^{12}$ L. Belloni, L'opera di Giambattista Morgagni: dalla strutturazione meccanica dell'organismo vivente all'anatomia patologica, in: Morgagni 4 (1971), pp.71-80; in Simposi Clinici 9 (1972), pp. I-VIII, ristampato nel mio volume Per la Storia della Medicina, Bologna 1980, pp.239-246; De la théorie atomistico-mécaniste à l'anatomie subtile (de Borelli à Malpighi) et de l'anatomie subtile à l'anatomie pathologique (de Malpighi à Morgagni), in: Clio Medica 6 (1971), pp.99-107; Giovanni Battista Morgagni (1682-1771) in der Geschichte der pathologischen Anatomie, in: Leopoldina - Mitteilungen der Deutschen Akademie der Naturforscher Leopoldina, Reihe 3, Jahrgang 18 (1972), 1975, pp. 103-112.

${ }^{13}$ R.Th. H.Laennec, Traité inédit sur l'anatomie pathologique. Préface par V.Cornil. Paris 1884, p. 37.

${ }^{14}$ M. Malpighi, Opere scelte, pp.427-429.

15 M. Malpighi, Opere scelte, p. 370 e tavola XX.

16 Bruno Zanobio, Le osservazioni microscopiche di Felice Fontana sulla struttura dei nervi, in: Physis 1 (1959), pp.307-320.

17 Bruno Zanobio, L'immagine filamentoso-reticolare nell'anatomia microscopica dal XVII al XIX secolo, in: Physis 2 (1960), pp.299-317; Micrographie illusoire et théories sur la structure de la matière vivante, in: Clio Medica 6 (1971), pp.25-40.

${ }^{18}$ R.Th. H.Laennec, Traité de l'auscultation médiate et des maladies des poumons et du cour. Edition de la Faculté de Médecine de Paris entièrement conforme à la seconde édition publiée en 1826. Paris 1879, p. 186.

19 Tale mi permetterei di continuare a considerare il Bichat, malgrado il recente lavoro di Othmar Keel, La généalogie de l'histopathologie. Une révision déchirante: Philippe Pinel, 
lecteur discret de J.-C.Smyth (1741-1821), Paris 1979, recensito da E. Ackerknecht in: Gesnerus 37 (1980), pp. 147-148.

${ }^{20} \mathrm{X}$. Bichat, Traité des membranes... Nouvelle édition, revue et augmentée de notes par M. Magendie, Paris 1827, p. 35 .

${ }^{21}$ L. Belloni, Auf dem Wege zur Gewebepathologie: 1769 erkennt Cotugno die schichteigenen Veränderungen der Haut im Verlaufe des Pockenprozesses, in: H.H.Eulner et al. (edit.) Medizingeschichte in unserer Zeit. Festschrift für Edith Heischkel und Walter Artelt zum 65. Geburtstag. Stuttgart 1971, pp. 245-258.

${ }^{22} \mathrm{X}$. Bichat, Anatomie générale appliquée à la physiologie et à la médecine, 1/1, Paris 1801, p. 1xxxviij.

\section{Zusammenfassung}

Die iatromechanische Lehre (Descartes, Borelli) und die mikroskopische Anatomie (Malpighi 1661) führten durch gegenseitige Befruchtung zur Entdeckung kleinster Maschinen in unserem Organismus. Die Krankheiten konnten demnach als Schädigungen dieser Maschinen aufgefaßt werden, d.h. als anatomisch erkennbare Organveränderungen. Es entstand daraus die von Morgagni im «De sedibus» (1761) systematisierte pathologische Anatomie, die sich ohne Verwendung des Mikroskopes entwickelte, da letzteres damals reichlich optische Täuschungen hervorbrachte. Aus demselben Grunde entstand auch die normale und pathologische Histologie ohne Anwendung des Mikroskops. Typisch dafür ist die Beobachtung der den einzelnen Hautschichten eigenen unterschiedlichen Veränderungen (Cotugno 1769) als Vorahnung der Begründung der Histologie (Bichat 1800/01).

\section{Summary}

The iatromechanical doctrine (Descartes, Borelli) and the microscopical anatomy (Malpighi 1661) brought about by mutual influence the discovery of smallest machines in our organism. The diseases could be understood as damages of these machines, i.e. as anatomically perceivable alterations of organs. Thus arose the pathological anatomy systematizised by Morgagni in "De sedibus" (1761) which developped without the use of the microscope (because of its many optical errors at that time). For the same reason the normal and pathological histology also arose without the use of the microscope. A typical example is the observation of the specific changes within the different separate strata of the skin (Cotugno 1769). This was an anticipation of the establishment of histology (Bichat 1800/01).

Prof. Dr. med. Luigi Belloni

Istituto di Storia della Medicina

Università degli Studi

Via Festa del Perdono, 7

I-20122 Milano 
